\title{
Key technologies of WSN remote monitoring system for Xinjiang coalfield fire
}

\author{
Qinghua Cao, Shu Yan \\ School of Computer Science and Communication Engineering \\ Jiangsu University \\ Zhenjiang, China \\ cqh@ujs.edu.cn
}

\author{
Guoqiang Xue \\ Institute of Geology and Geophysics \\ Chinese Academy of Science \\ Beijing, China \\ ppxueguoqiang@163.com
}

\begin{abstract}
For the real-time, on-site, direct monitoring of coalfield fire area temperature, we developed a permanent WSN monitoring system with the functions of continuous collection, high temperature detection, remote control, etc. By designing the software and hardware of SPI interface between the JN5139 chip and the MAX31855 with $K$ type thermocouple, we developed a new type of high temperature sensor node, which temperature range extended to $-40^{\circ} \mathrm{C} \sim 1300^{\circ} \mathrm{C}$ from standard WSN node temperature range. This node can be placed directly on the underground burned area roadway for on-site monitoring. The solar power supply device with protective circuit and low power consumption peripheral circuit provide the data acquisition network with a sustainable energy for all-weather and real-time monitoring. In the uplink data transmission through mobile communication network and Internet to the monitoring center, the heartbeat packets mechanism without manual intervention was used to automatic reconnection, which keeps the link unobstructed to realize the remote control monitoring. The monitoring system that deployed in 12 fire areas of Xinjiang has worked for $\mathbf{4 5}$ mouths, and that means technical measures used can guarantee the long-term stability operation of the monitoring system without maintenance. Urumqi monitoring center has received more than $\mathbf{1 1 0}$ ten thousand field information that can be preserved permanently and queried freely after authorized. This information provides basic data for the management of large area of coal spontaneous combustion fire area in Xinjiang and for governance results defend.
\end{abstract}

Keywords-WSN;real-time; high temperature; bidirectional communication; remote control; heartbeat package

\section{INTRODUCTION}

Xinjiang coalfield is shallow buried and mostly expose. During the coalfield fire extinguishing and managing process, a remote and long-term monitoring method for real-time and direct temperature measurement is also needed in addition to the manual inspection, airborne observation ${ }^{[1]}$ and satellite monitoring ${ }^{[2]}$. The modern wireless sensor network (WSN) technology makes it possible ${ }^{[3]}$. The WSN fire area monitoring system throughout Xinjiang is different from WSN that is used for the environment, human health, city, structure, agricultural, coal mine and landslides monitoring ${ }^{[4]-[9]}$. There are many special questions should be solved: measurement of fire area temperature that exceed the range of a standard sensor for general nodes, continuous energy supply for permanent WSN nodes, automatic connection of communication link in remote data transmission without manual intervention, etc. 2011 till now, the WSN monitoring system that was deployed in 12 fire area of Xinjiang, such as Songshutou, Daquanhu and Kueraken, has been working for 45 months. Battery electricity was full, communication link was unblocked, observation data returned from the monitoring area was complete and reasonable, and control instruction sent to monitoring area was executed correctly.

\section{Structure of XinJiang WSN Remote Fire MONitoring SYSTEM}

The on-site data collection network that consists of WSN nodes deployed in fire areas, the remote transmission system and monitoring center server constituted a complete WSN monitoring system (Fig. 1). In the uplink from on-site data collection network to monitoring central server, field data that was collected by high temperature nodes was wirelessly transmitted to sink node and then reached to the server in Urumqi through GPRS, mobile system and Internet. In the downlink from monitoring to on-site network, control information was transmitted from server to field nodes.

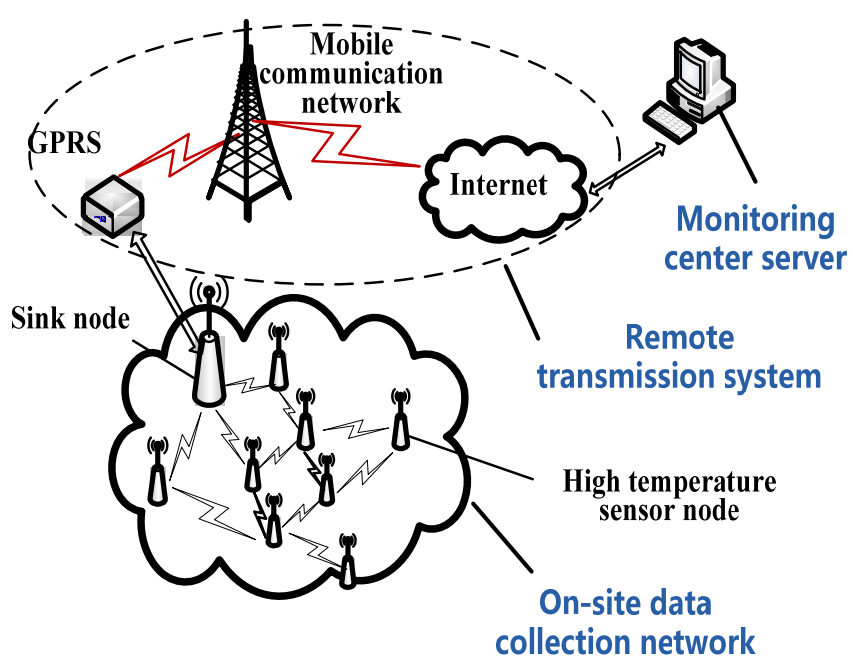

Fig. 1. Structure of Xinjiang WSN remote monitoring system. 


\section{High TEMPERATURE SENSOR NODE}

In order to measure the fire area temperature from tens ${ }^{\circ} \mathrm{C}$ below zero in winter to thousand ${ }^{\circ} \mathrm{C}$ during coal combustion, we used a K-type thermocouple with measurement range of $-40^{\circ} \mathrm{C} \sim 1300^{\circ} \mathrm{C}$ as temperature sensor. As shown in Fig. 2a, the positive and negative electrodes of the thermocouple respectively connect with the $\mathrm{T}+$ and $\mathrm{T}$ - of MAX31855 (a thermocouple digital temperature sensor chip). The measured temperature signal in the form of analog voltage was transferred to MAX31855 and the signal attenuation errors because of transmission could be compensated by the collection software programming of node. The main control unit JN5139 connected with the slave chip MAX31855 through the three-wire system SPI. The first line between SCLK and SCK offered clock signal, the second line between DIO0 and $\overline{\mathrm{CS}}$ selected slave chip, the third line between MIMO and SO of received digital data. The flow chart of thermometry control program written in JN5139 chip is shown in Fig. 2b.

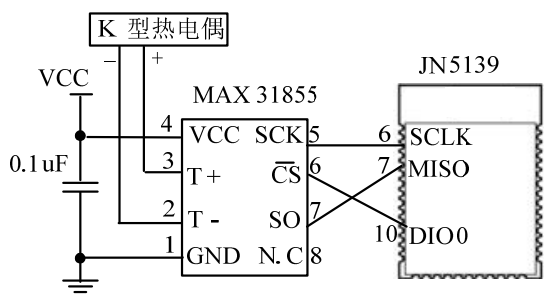

(a)

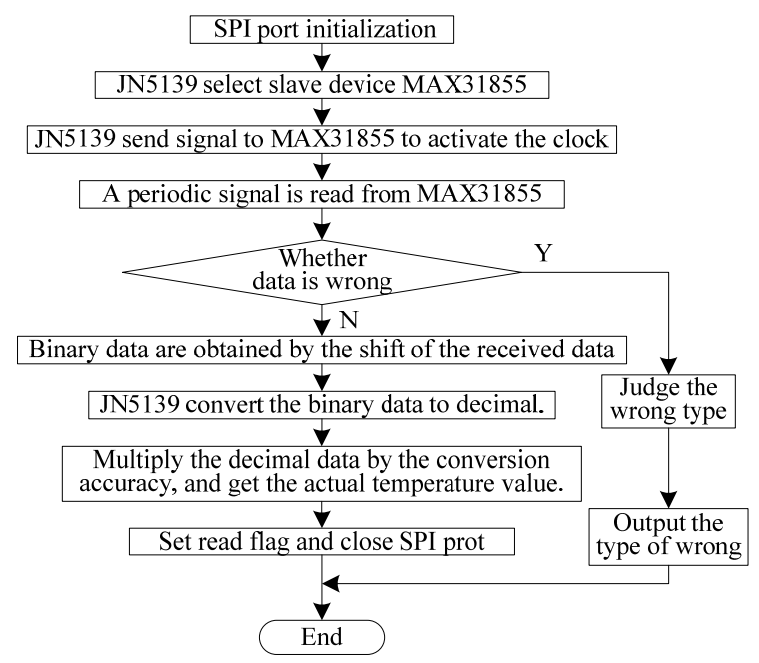

(b)

Fig. 2. The hardware connection and software flow chart of high temperature sensor node. (a) Connection diagram of JN5139 chip, MAX31855 and K-type thermocouple. (b) The flow chart of thermometry control software.

First step was SPI initialization such as setting the quantity of SPI equipment, the first bit of data when transmit, the clock rate and trigger edge, interrupt and automatic chip selection. In accordance with the MAX31855 SPI operating time sequence, JN5139 sent CS signal to select MAX31855 and offered clock signal. JN5139 read the 32 bits data and judged whether it is wrong. If wrong, judge the type of error. If right, data was shifted, changed and converted to readable decimal data. Finally, the read flag was set and the SPI port closed. Next time when you need to read the data, the above steps should be repeated again. The compiled program was written in the JN5139 chip as the main unit.

\section{NETWORKING UNINTERRUPTED COLLECTION TECHNOLOGY}

The continuous energy of permanent on-site monitoring network was provided by solar power supply device. Because the remote monitoring system had higher request for free maintenance, we designed a protection circuit that prevents the excessive discharging of the battery for extending the maintenance cycle of solar power supply device.

As shown in Fig. 3a, when the sunshine was enough, the charging protection circuit made solar panel charge to battery and directly supply power to the node through the voltage stabilization circuit in Fig. 3b. When the sunshine was not enough or in the night, if the solar panel output voltage was lower than the battery voltage, the transistors Q1 reversely blocked and then node turned to be powered by the battery. This circuit was constant-voltage charging. Three-terminal voltage regulator TL431 and adjustable resistance W1 formed voltage regulation circuit that prevented the battery from overshoot by setting the charging threshold. With the increasing voltage of the battery, the charging current decreased gradually to the trickle charging in order to ensure the battery do not overcharge. According to the power self consumption of battery, the trickle circuit value was set by the transistor Q2 and the adjustable resistance W2.

As shown in Fig. 3b, the DC-DC circuit consisting of voltage regulator module HT7330 and capacitances C1 C4 also acted as discharging protection circuit with simple structure and low power consumption $(0.3 \mathrm{~mA})$. When the input (pin 1) voltage of HT7330 was greater than or equals to the battery voltage protection threshold (3.2V), the output (pin 2) voltage stabled at $3 \mathrm{~V}$ and supplied power to node. During the battery voltage downed to $2.7 \mathrm{~V}$, HT7330 output voltage gradually decreased from $3 \mathrm{~V}$ to stop working, and output current reduced to microampere level for discharging protection. The small capacitors C1 and C3 filtered out high frequency noise and impulse interference, and the large capacitors C2 and C4 filtered out low frequency noise.

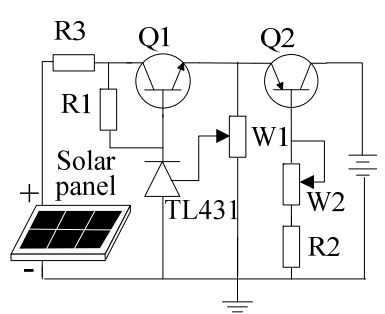

(a)

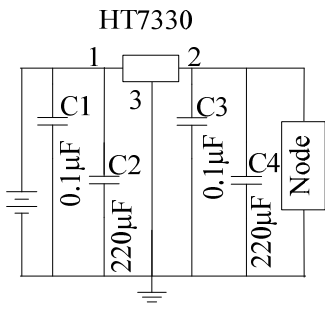

(b)
Fig. 3. The charging and discharging protection circuit of solar power. (a) Charging protection circuit. (b) Discharging protection circuit. 
The average daily energy consumption of high temperature sensor node data acquisition and transmission is $0.126 \mathrm{Ah}$. In order to make the solar power supply device have plenty of energy storage during the continuous overcast day, rain, fog, snow and winter sunshine lack, etc., we equipped sensor node with a 3.2V 4Ah battery that can be charged full about 6 hours (less than a day) by $700 \mathrm{~mA}$ current of a 5W 6V solar panel. In this paper, we selected a lithium iron phosphate battery in order to fit the $50{ }^{\circ} \mathrm{C}$ summer high temperature and near $-40{ }^{\circ} \mathrm{C}$ winter low temperature in the wilderness. With charging and discharging protection circuit shown in Fig. 3, the solar power supply device, which was filled once, can maintained node working about 30 days, enough to cope with the extreme weather conditions for a long time and make the whole network realize uninterrupted collection.

\section{BIDIRECTIONAL COMMUNICATION AND REMOTE CONTROL TECHNOLOGY}

\section{A. Heartbeat Package Mechanism}

By GPRS module installed on the sink node, field monitoring data are transparently transferred in the form of IP packet following TCP/IP protocol, and finally arrived to the server through mobile network and Internet. With the rapid development of mobile business, network congestion and accidental disconnection happen occasionally. How to make the GPRS module in the wilderness restore the communication without manual intervention and ensure the reliable transmission of the data is an important problem to be solved in the remote communication. To this end, we used the heartbeat packet technology [10], a data packet transmitted by GPRS module in certain frequency, to occupy channel and keep the link unblocked. If communication was unexpectedly interrupted, the link should automatically resume by the heartbeat packets. Data length of the sub package also is an important parameter of GPRS communication. A too large package is prone to lose, and a too small data packet adds a meaningless overhead. From the heartbeat package configuration interface of GPRS module, we set the heartbeat cycle to 60s and set the data package length to 300 bytes. Test result shown that the packet loss rate of transfer data reduce from maximum $7 \%$ without the heartbeat packets to $0.31 \%$ with heartbeat package.

\section{B. Bidirectional Communication Protocol}

The bidirectional communication protocol for uplink and downlink includes: unit format, content, the meaning and the send-receive sequence of data for correct transmission and analysis.

TABLE I lists the data package format with 16 byes including 6 bytes packet header and 10 bytes data field in uplink. The main fire area number is the label of field fire area, occupying 1 byte. In one main fire area, there could be deployed many sub fire areas, each of which have the only GPRS. Life and security field each own 1 byte, reserved for building network later. Data field is filled by the actual monitoring data.

TABLE II lists the data package format of downlink. The type flag field with 1 byte represents the type of control information. For example, "0" mean network clock correction and " 1 " mean sample interval modification. The type flag field could be set a maximum of 255 types of control, which reserved extended space for later development. The size of the control information field, filled with the specific control data, could be set variable value between 1 byte to 12 bytes. The end flag with 1 byte, filled with character " $E$ ", indicate the end of data package.

TABLE I. DATA PACKAgE Format OF UPLINK

\begin{tabular}{|c|c|c|c|c|c|c|c|c|c|c|}
\hline \multirow{3}{*}{ Field } & \multicolumn{9}{|c|}{ Header } & \multicolumn{5}{c|}{ Data } \\
\cline { 2 - 11 } & $\begin{array}{c}\text { Main fire area } \\
\text { No. }\end{array}$ & $\begin{array}{c}\text { Child fire } \\
\text { area No. }\end{array}$ & $\begin{array}{c}\text { Node } \\
\text { No. }\end{array}$ & Lifetime & Safety & $\begin{array}{c}\text { Ambient } \\
\text { temperature }\end{array}$ & $\begin{array}{c}\text { Ambient } \\
\text { humidity }\end{array}$ & $\begin{array}{c}\text { Fire area } \\
\text { temperature }\end{array}$ & $\begin{array}{c}\text { Supply } \\
\text { voltage }\end{array}$ & $\begin{array}{c}\text { Battery } \\
\text { voltage }\end{array}$ \\
\hline Bytes & 1 & 1 & 2 & 1 & 1 & 2 & 2 & 2 & 2 & 2 \\
\hline
\end{tabular}

TABLE II. DATA PACKAgE Format OF DOWNLINK

\begin{tabular}{|c|c|c|c|}
\hline Field & Type flag & Control data & End flag(E) \\
\hline Bytes & 1 & $1 \sim 12$ & 1 \\
\hline
\end{tabular}

\section{Remote Control}

Through the control information sent by the down link, users could adjust the parameter of on-site network at serverside. Server sent the control instruction along with ACK message to the on-site network after received the field data. The initial value of data sampling interval was 1 hour. The authorization user analyzed the received data of server, and then adjusted the sampling interval within the range of 1 hour to 24 hours according to the change rate of fire area ground temperature ${ }^{[11]}$. If not to be adjusted, the sampling interval values in instruction keep unchanged. Authorized users also could send timing command to monitoring site. Downlink control command was analyzed by the sink node, and then was forwarded to high temperature monitoring nodes for performing together.

\section{SySTEM OpERATION RESUltS}

The whole Xinjiang fire area monitoring system could contain 255 main fire areas at most, and each main fire area included 255 sub fire areas at most. Up to now, the monitoring networks have been deployed in 12 main fire areas such as Songshutou in northern Xingjiang, Kueraken in southern 
Xingjiang, etc. Each subnet (e.g. Songshutou shown in Fig. 4) consisted of one sink node and 4 10 high temperature sensor nodes. GPRS module on the sink node transmitted monitoring data to the monitoring center at Xinjiang Coal Field FireFighting Engineering Bureau server in Urumqi, via mobile network and Internet.

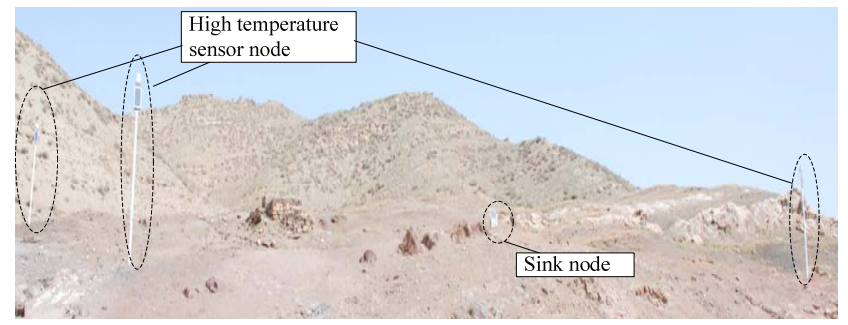

(a)

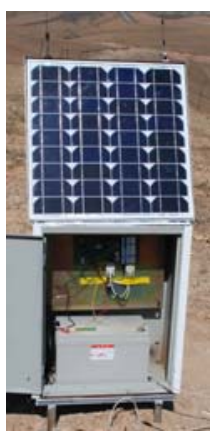

(b)

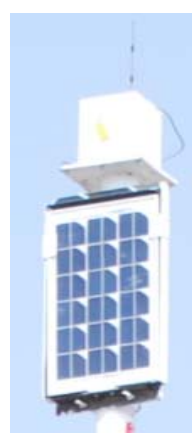

(c)
Fig. 4. (a)The WSN monitoring system deployed in Songshutou fire area (b) Sink node (c) High temperature node.

\section{CONCLUSIONS}

The WSN remote monitoring system provided a real-time, on-site and direct temperature measured monitoring method for Xinjiang Coalfield Fire Engineering Bureau to autonomously access, addition to the manual detection, aviation and satellite remote sensing monitoring. Due to the designed high temperature sensor node, the temperature measurement range of monitoring system extended to $-40^{\circ} \mathrm{C}$ $\sim 1300^{\circ} \mathrm{C}$ from range of WSN standard temperature sensor. This temperature range covered the coal seam temperature changing range from the combustion of accumulated temperature to the flameout after governance and in winter.
Until now, Xinjiang fire area remote WSN monitoring system has been running for three years and nine months and has collected data more than 1,100,000. All of these data and subsequent data were stored in Urumqi monitoring center server, and provide the basis for fire area governance and management. In the process of forming the whole Xinjiang WSN system in the future, GPRS will be upgraded due to the development of 4G mobile network. More information such as gas, image and video will be observed and transmitted. Xinjiang coalfield will be placed inside a full range, allweather continuous monitoring.

\section{REFERENCES}

[1] L.F. Radke, T.L. Clark, J.L. Coen, C.A. Walther, and R.N. Lockwoodet, "The WildFire Experiment (WiFE): Observations with airborne remote sensors”, Canadian Journal of Remote Sensing, 26(5), 406-417,2000.

[2] S.A. Christopher, P. Gupta, U. Nair, "Satellite remote sensing and mesoscale modeling of the 2007 Georgia/Florida fires", IEEE Journal of Selected Topics in Applied Earth Observations and Remote Sensing, 2(3), 163-175, 2009.

[3] D. Estrin, R. Govindan, J. Jeidemann, “Next century challenges: Scalable coordinate in sensor network", Proc 5th ACM/IEEE Int'l Conf on Mobile Computing and Networking, 263-270, 1999.

[4] B. Kerkez, S. Glaser, R.C. Bales, M. W. Meadows, "Design and performance of a wireless sensor network for catchment-scale snow and soil moisture measurements” Water Resources Research, 48(9), 1-18, 2012.

[5] G. Blumrosen, M. Uziel, B. Rubinsky, D. Porrat, "Tremor acquisition system based on UWB wireless sensor network”, 2010 International Conference on Body Sensor Networks, 187-193, 2010.

[6] T. Watteyne, K.S.J. Pister, "Smarter cities through standards-based wireless sensor networks”, IBM Journal of Research and Development, 55(1-2), 7:1-7:10, 2011.

[7] S.N. Pakzad, G.L. Fenves, S.Kim, D.E. Culler, "Design and implementation of scalable wireless sensor network for structural monitoring”, Journal of Infrastructure Systems, 14(1), 89-101, 2008.

[8] F.J. Pierce, T.V. Elliott, "Regional and on-farm wireless sensor networks for agricultural systems in Eastern Washington”, Computers and Electronics in Agriculture, 61(1), 32-43, 2008.

[9] M.V. Ramesh, "Design, development, and deployment of a wireless sensor network for detection of landslides", Ad Hoc Networks, 13(1), 2-18, 2014.

[10] A. Striegel, G. Manimaran, "Edge-based fault detection in a DiffServ network", Proceedings of the 2002 International Conference on Dependable Systems and Networks, 79-88, 2002.

[11] Q.H. Cao, S. Yan, N. Zhu, "Synchronization recovery mechanism of long-time and deep sleep wireless sensor network", CN 102821446 A, 2012-12-12. 